\title{
A study of Coeliccia cyanomelas Ris, 1912 (Odonata: Platycnemididiae)
}

\author{
Xin $\mathrm{Yu}^{\mathrm{a} *}$, Min Zhang ${ }^{\mathrm{a}}$ and Xin Ning ${ }^{\mathrm{b}}$ \\ ${ }^{a}$ College of Life Sciences, Chongqing Normal University, Chongqing, PR China; ${ }^{b}$ Institute of Entomology, \\ College of Life Sciences, Nankai University, Tianjin, PR China
}

(Received 16 March 2019; accepted 7 July 2019)

\begin{abstract}
Coeliccia cyanomelas is studied based on a large series of specimens from its whole distribution range. Intraspecies variations of color patterns on the thorax are discussed. Coeliccia sexmaculata Wang, 1994, Coeliccia mingxiensis Xu, 2006, and Coeliccia wilsoni Zhang \& Huo, 2011 are all assigned as junior synonyms of Coeliccia cyanomelas. One ‘variety' from Guizhou is reported and discussed briefly.
\end{abstract}

Keywords: Zygoptera; color pattern variation; new synonyms; new variety; dragonfly

\section{Introduction}

The oriental genus Coeliccia Kirby, 1890, with 73 recorded species globally, is the second largest genus in the family Platycnemididae (Schorr, Lindeboom, \& Paulson, 2009). A total of 11 Coeliccia species have been recorded from China, mainly distributed in Yunnan and Guangxi provinces (Yu, 2010). They are Coeliccia ambigua Asahina, 1997; Coeliccia chromothorax (Selys, 1891); Coeliccia cyanomelas Ris, 1912; Coeliccia didyma (Selys, 1863); Coeliccia flavicauda Ris, 1912; Coeliccia galbina Wilson and Reels, 2003; Coeliccia loogali Fraser, 1932; Coeliccia poungyi Fraser, 1924; Coeliccia satoi Asahina, 1997; Coeliccia scutellum Laidlaw, 1932 and Coeliccia sexmaculata Wang, 1994. Among them, C. cyanomelas is the most widespread species, with a distribution covering the whole of south China and extending to Vietnam (Steinhoff \& Uhl, 2015; Yu, 2010). Studies have shown that the male of $C$. cyanomelas presents a large variation in body color pattern (Yu, 2008; Steinhoff \& Uhl, 2015). Three recently proposed species from China (Wang, 1994; Xu, 2006; Zhang \& Huo, 2011) were thought to be very similar to $C$. cyanomelas and therefore need further study.

C. didyma (Selys, 1863) resembles $C$. cyanomelas in appearance, but is distributed further west (India, Peninsula Malaysia, and Thailand). Both Fraser (1933) and Asahina (1984) have mentioned that the first type specimen of $C$. didyma that Selys studied is from Tibet. Unfortunately, the abdomen end of this specimen is lost. Asahina (1984, p. 4) added that "This looks rather different bluish species resembled to C. cyanomelas Ris (Taiwan) with four pale spots on the front of pterothorax." He then cited Laidlaw's comment that Selys might have confused Tibet with Assam. Nobody knows why Asahina did not check the genital ligula of that specimen. The

*Corresponding author. Email: lannysummer@163.com 


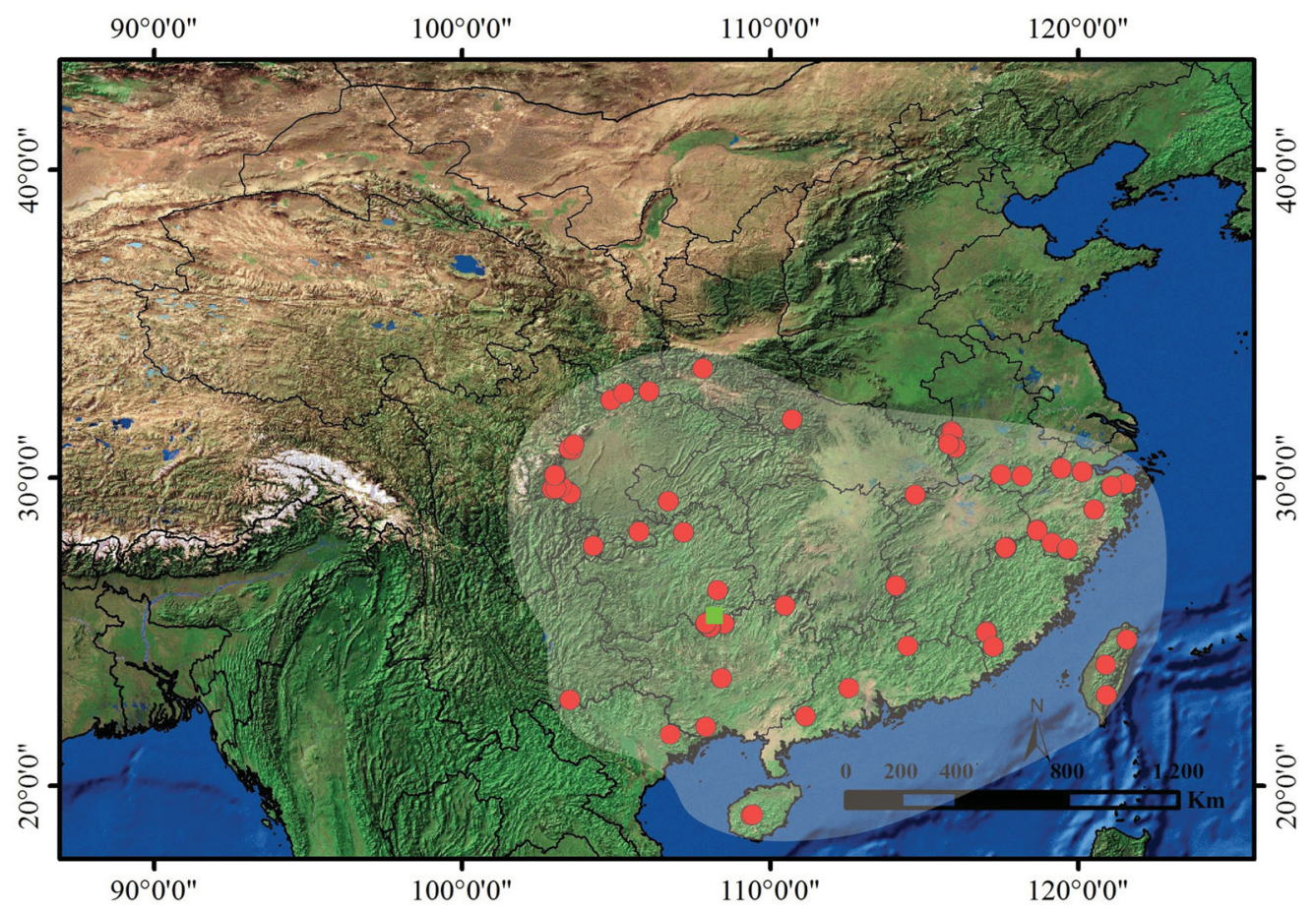

Figure 1. Map of the distribution of C. cyanomelas. Red circles indicate the localities of specimens, the green square indicates the locality of the new variety, and the gray shading shows the whole distribution range.

shape of the ligula in C. cyanomelas and C. didyma is completely different. We now can confirm that $C$. cyanomelas occurs in west Sichuan (Figure 1), an area used to be called Tibet (Yu \& Bu, 2009). There are now no confirmed records of $C$. didyma from China. In any case, the didyma group (including C. didyma, C. cyanomelas, and maybe others) deserves a further study to test if the similar color pattern is phylogenetically meaningful.

The present study focused on the color variation of $C$. cyanomelas, synonymized three species, and reported a new 'variety'.

\section{Material and methods}

Specimens were collected from China (Anhui, Chongqing, Fujian, Gansu, Guangdong, Guangxi, Guizhou, Hainan, Henan, Hubei, Hunan, Jiangxi, Shaanxi, Sichuan, Taiwan, Yunnan, and Zhejiang) and Vietnam from 1982 to 2016, and were either kept in alcohol or dried, and stored in College of Life Sciences of Chongqing Normal University. All the living photos were taken in the field with digital cameras Canon Power Shot A710 (Zhuhai, China) or Nikon D3200 (Bangkok, Thailand). Character photos were taken in the laboratory using Zeiss V20 (Jena, Germany) microphotography system. Specimens were examined and dissected under a Zeiss V8 stereomicroscope.

\section{Taxonomy}

Coeliccia cyanomelas Ris, 1912 


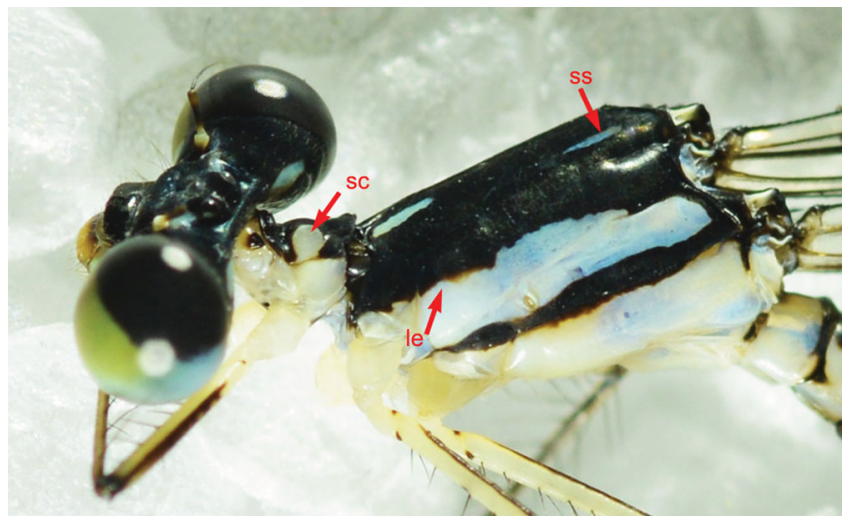

Figure 2. Head and thorax of C. cyanomelas. Abbreviations: sc, pale circular marks on the dorsum of middle lobe of pronotum; ss, pair of small stripes on the upper side of synthorax dorsum; le, lower edge of the black area on dorsal synthorax.

Coeliccia cyanomelas Ris, 1912: 66, Formosa; Needham, 1930: 247, pl. XVI:12; Asahina, 1956: 204-228; Asahina, 1961:10, figs 55, 56; Sui \& Sun,1986: 280; Yu, 2008: 2 (10), pl. V: 8-10; Steinhoff \& Uhl, 2015: 257-276, figs 8b, c, $9,10,11 \mathrm{~d}-\mathrm{f}$.

Coeliccia sexmaculata Wang, 1994: 82-84, 1234-5. syn. nov.

Coeliccia mingxiensis Xu, 2006: 251-255, 1, pl. VII: b. syn. nov.

Coeliccia wilsoni Zhang \& Huo, 2011: 51-56, 1-9. syn. nov.

\section{Material}

One male (Holotype of C. sexmaculata), Luanchuan, Henan, 20 August 1986, Xiujiang Zhang leg. 1 male, 1 female (paratypes of $C$. sexmaculata), ditto. 3 males, 2 female, Yuanchong, Jinzhai, Anhui, 9 June 2012, Xin Yu leg.; 3 males, 1 female, Huangshan, Anhui, 5 May 2014, Xin Yu leg.; 6 males, 7 females, Banan, Chongqing, 8 July 2016, Junli Xue leg.; 7 males, 3 females, Wuyishan, Fujian, 5 May 2014, Xin Yu leg.; 15 males, 5 females, Nanping, ditto., 510 August 2014, Xin Yu leg.; 3 males, 6 females, Zhangzhou, ditto., 13-15 August 2014, Junli Xue leg.; 6 males, Longyan, ditto., 5 August 2016, Xin Yu leg.; 3 males, 3 females, Zhangzhou, ditto., 15 August 2014, ditto.; 4 males, 1 female, Bikou, Wenxian, Gansu, 13 July 2005, ditto.; 3 male, Dinghushan, Guangdong, 26 July 2009, Ying Cui leg.; 2 male, 1 female, Maoming, ditto., 1 August 2009, Min Li leg.; 3 male, Leye, Guangxi, 24 July 2004, Xin Yu leg.; 3 males, 1 female, Damingshan, ditto., 9 August 2011, ditto.; 3 males, 1 females, Xingan, ditto., 9 July 2009, Kai Dang leg.; 19 males, 5 females, Mulun, ditto., 21 July 2015, Xin Yu leg.; 4 males, 2 females, Huaxi, Guizhou, 5 August, ditto.; 5 males, females, Zunyi, ditto., 6 August 2013, Xiurong Li leg.; 6 males, 1 female, Maolan, ditto., 25 July 2015, Xin Yu leg.; 11 males, 1 female, Wengang, Libo, ditto., 25 July 2015, ditto.; 1 male, Leigongshan, ditto., 1 August 2015, Junli Xue leg.; 1 male, 1 female, Diaoluoshan, Hainan, 31 May 2007, Xin Yu leg.; 2 males, Bawangling, ditto., 750 m, 9 June 2007, ditto.; 2 males, Yinggeling, Baisha, ditto., 1 August 2013, Jie Yang leg.; 3 males, 1 females, Neixiang, Henan, 30 May 1958, Zhiguo Wang leg.; 2 males, 2 females, Shangcheng, ditto., 20 June 1986, Jianguang Xiao leg.; 10 males, 1 female, Luanchuan, Luoyang, ditto., 3 July 2017, Xin Ning leg.; 6 males, 3 females, Huanggang, Hubei, 2 July 2014, Xin Ning leg.; 7 males, 4 females, Shiyan, ditto., 14 July 2014, Junlixue leg.; 3 males, Xianning, ditto., 1 August 2010, Wenjun BU leg.; 5 males, 1 females, Zhangjiajie, Hunan, 6 August 2015, Xin Yu 
$158 X . Y u$ et al.

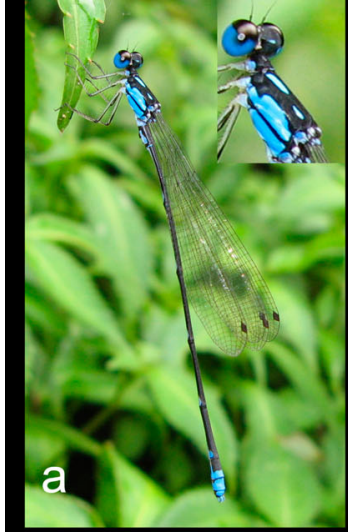

Damingshan, Guangxi

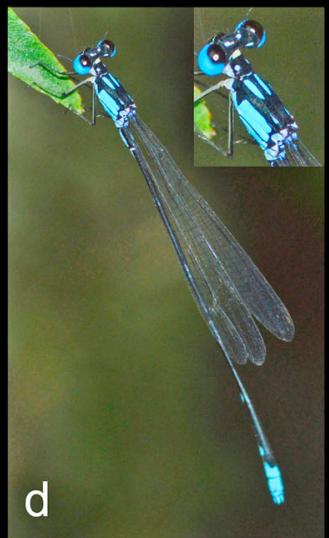

Hechi, Guangxi

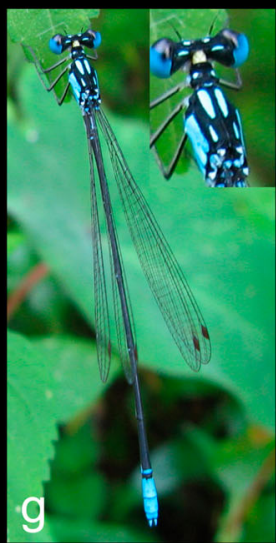

Yaan, Sichuan

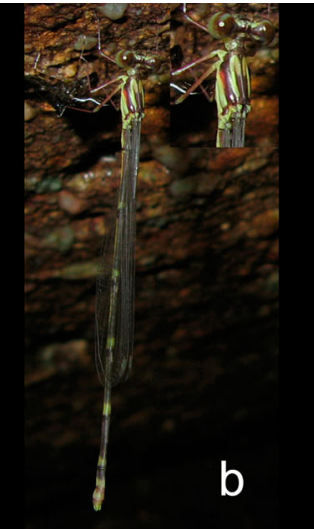

Damingshan, Guangxi

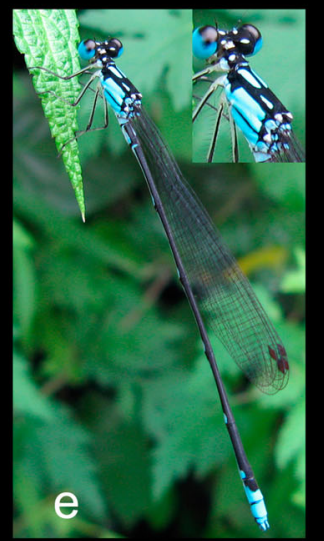

Zunyi, Guizhou

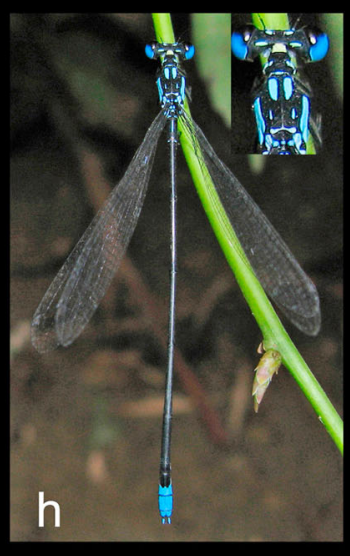

Qingchengshan, Sichuan

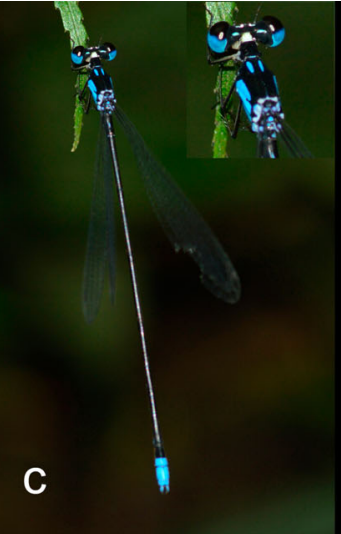

Hechi, Guangxi

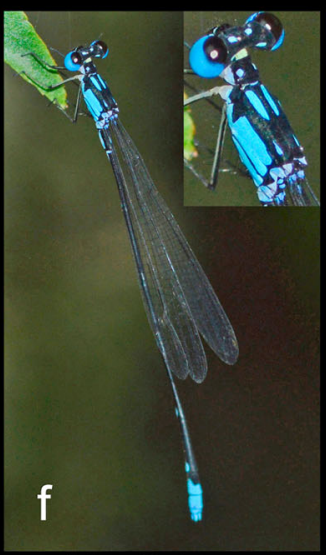

Libo, Guizhou

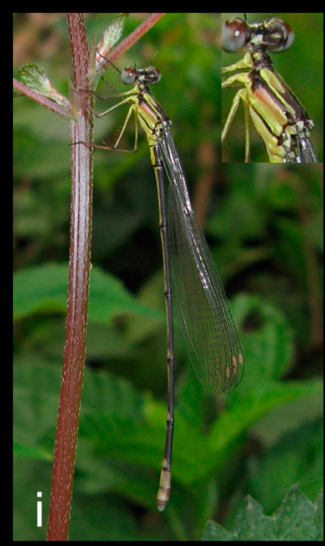

Emeishan, Sichuan

Figure 3. Field habitus photos of C. cyanomelas from different populations (1). 


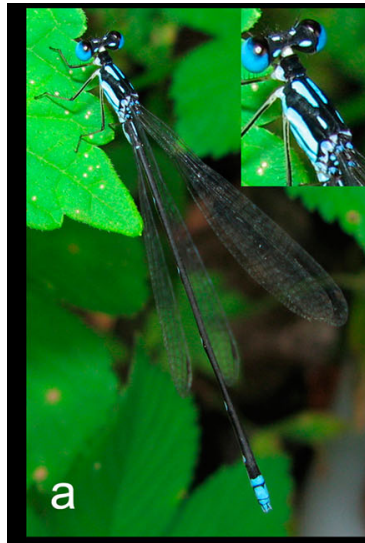

Tianmushan, Zhejiang

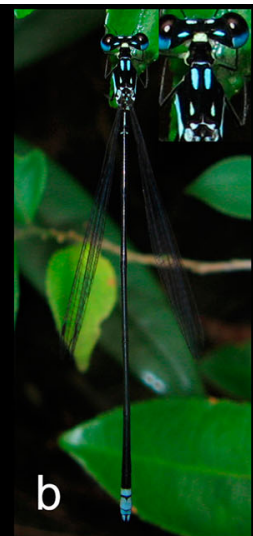

Taishun, Zhejiang
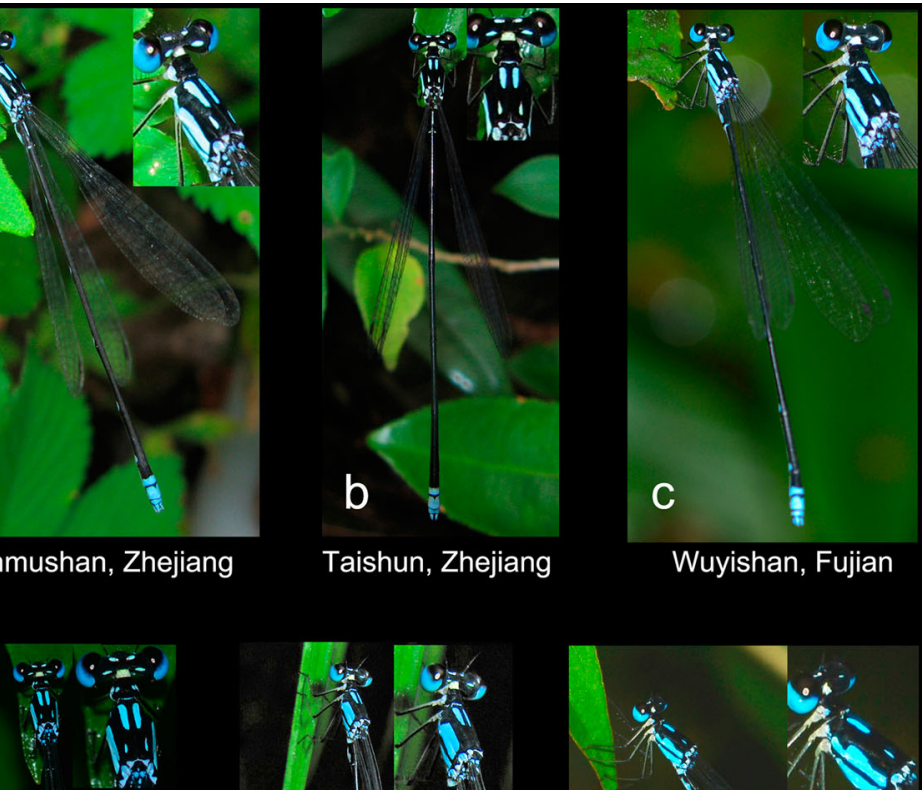

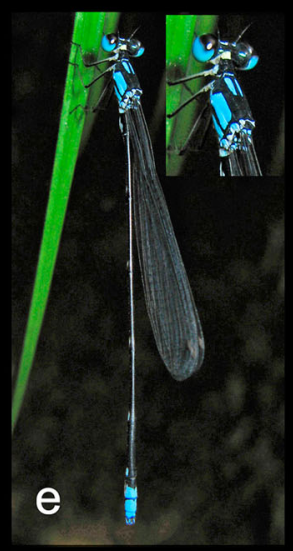

Hainan
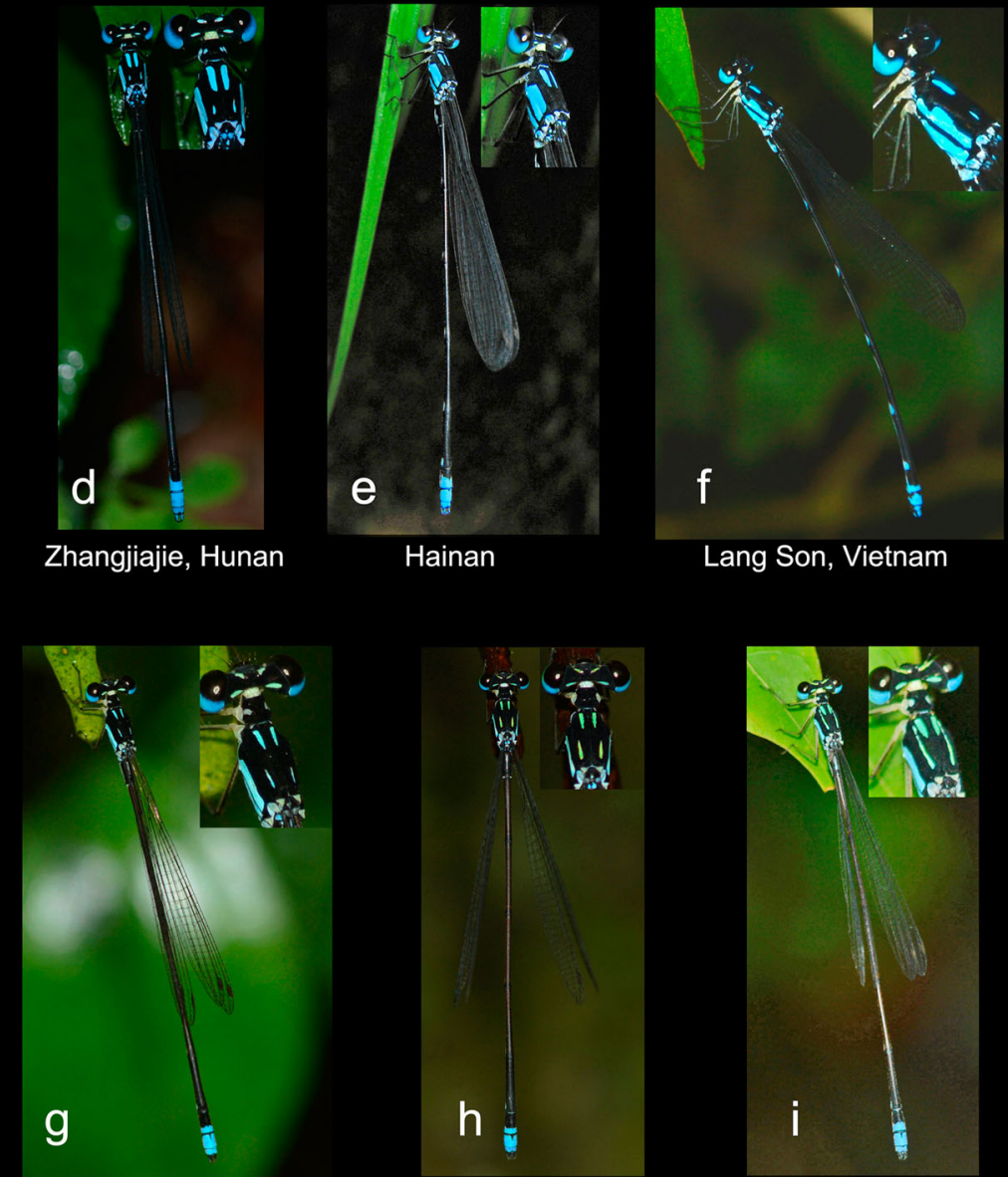

Libo, Guizhou

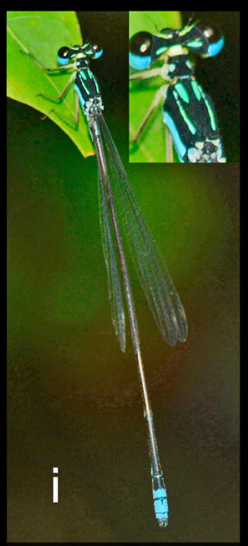

Libo, Guizhou

Figure 4. Field habitus photos of C. cyanomelas from different populations (2). 

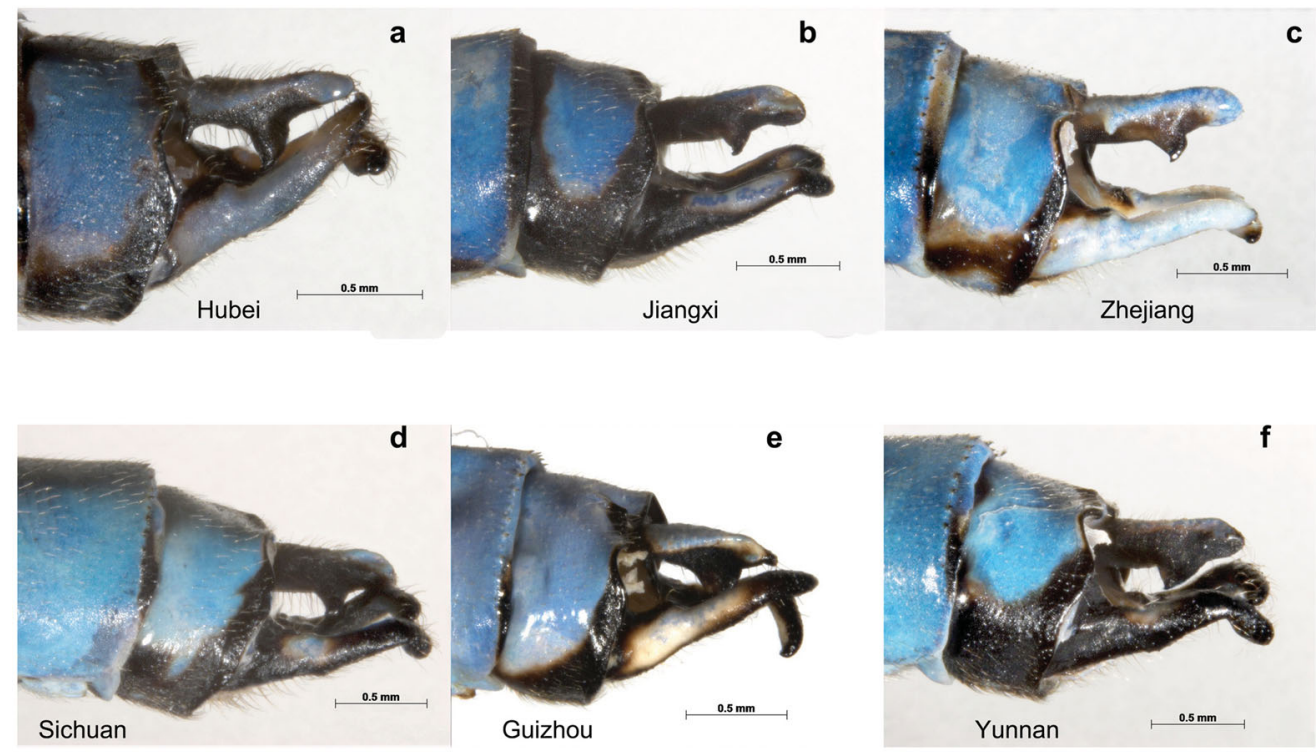

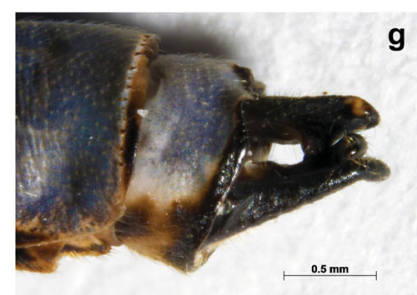

Guizhou

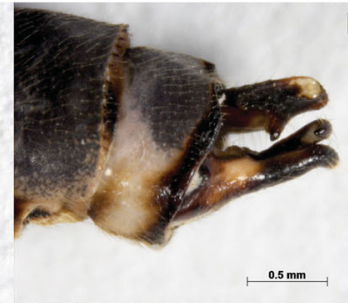

Guizhou h

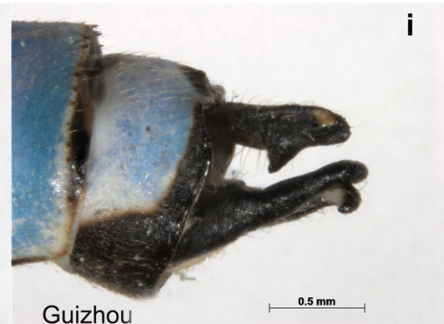

Figure 5. Lateral view of caudal appendages of $C$. cyanomelas with the left cerci removed.

leg.; 2 males, 1 female, Yifeng, Jiangxi, 9 June 2004, Xin Yu leg.; 7males, 3 females, Jinggangshan, ditto., 19 August 2016, ditto.; 1 male, Foping Shaanxi, 27 July 2013, Huaxi Liu leg.; 15 males, 6 female, Nanzheng, Hanzhong, ditto., 10 July 2017, Xin Ning leg.; 8 males, Emeishan, Sichuan, 18 August 2013, Xin Yu leg.; 6 males, 3 females, Guangyuan, ditto., 19 July 2016, Junli Xue leg.; 4 males, Yaan, ditto., 25 July 2011, Xin Yu leg.; 2 males, 1 female, Nantou, Taiwan, 6 November 2016, Huaxi Liu leg.; 5 males, Gaoxiong, ditto., 7 November 2016, ditto.; 9 males, 1 female, Zhaotong, Yunnan, 4 July 2016, Junli Xue leg.; 4 males, 1 female, Tianmushan, Zhejiang, Xin Yu leg.; 7 males, Hangzhou, ditto., 21 August 2014, Junli Xue leg.; 4 males, 1 female, Jinhua, ditto., 29 July 2015, Wenbo Yi leg.; 5 males, 3 females, Ningbo, ditto., 1 August 2016, Xin Yu leg.; 4 males, 2 females, Quzhou, ditto., 9 August 2016, ditto.; 4 males, Mau Son Mt., Loc Binh, Lang Son, Vietnam, 21 May 2015, ditto.

\section{Remarks}

According to our study, C. cyanomelas occurs in Anhui, Chongqing, Fujian, Gansu, Guangdong, Guangxi, Guizhou, Hainan, Henan, Hubei, Hunan, Jiangxi, Shaanxi, Sichuan, Taiwan, Yunnan, Zhejiang of China, and north Vietnam (Figure 1). Male color pattern of this species, especially on the thorax, shows large variations, which existed not only between but also within geographic populations. These variations may have led to a series of taxonomic mistakes (Yu, 2008). The 


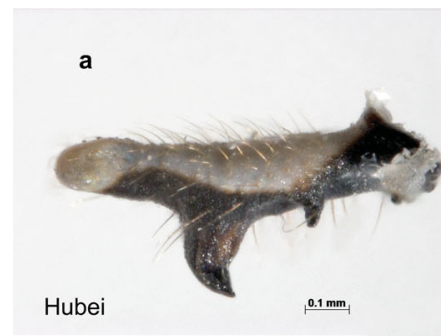

d
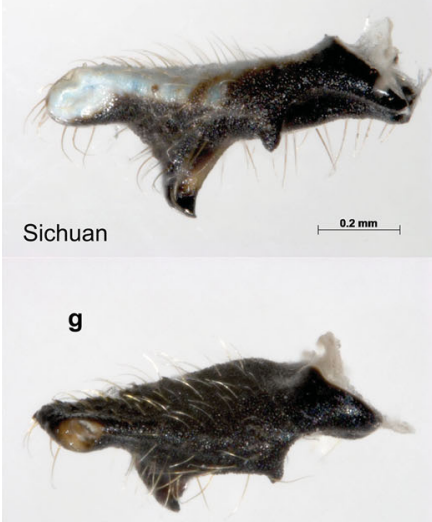

Guizhou

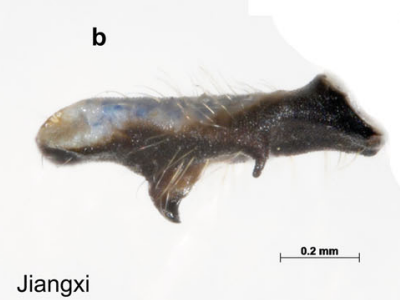

e
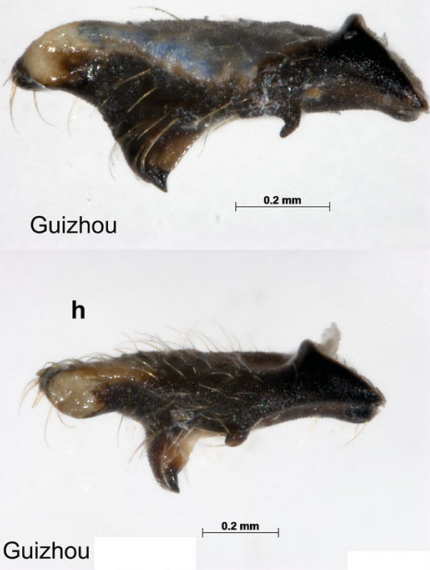
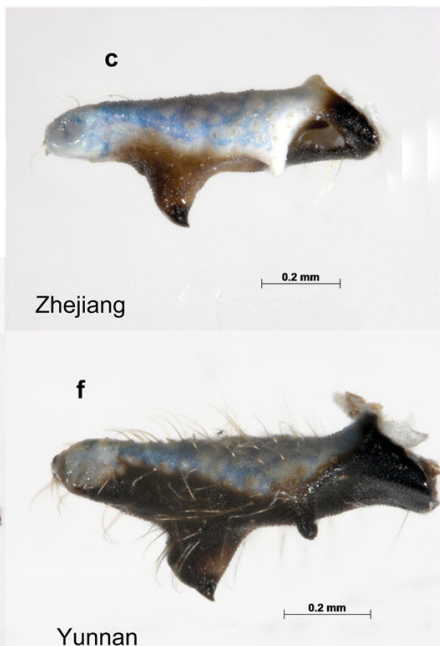

Yunnan

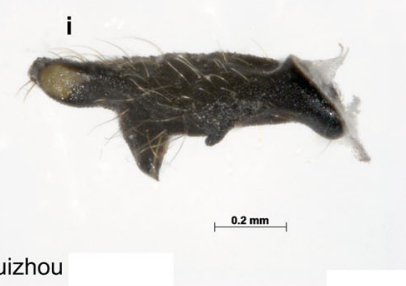

Figure 6. Dissected left cerci of C. cyanomelas.

major variations include: (1) the presence or absence of a pair of pale circular marks on dorsum of middle lobe of pronotum (SC); (2) the presence or absence, as well as the relative size of a pair of small stripes at upper side of synthorax dorsum (SS); and (3) the shape of the lower edge of the black area on dorsal synthorax (LE) (Figure 2). SC has been used as an important diagnostic character in Wang (1994) and Xu (2006). However, it is not a creditable feature since the SC can either present or absent in individuals even within one population (Figure 3c, d). There is a similar situation for SS, which shows continuous variations from absent to quite large in Sichuan and Guangxi populations at least (Figure 3c, d). Few researchers previously have noted the variation of LE, although it is obvious. LE can be relative smooth like a straight line (Figures 2, 4a) or zigzag (Figure 3d-f) in different populations.

\section{Synonymization}

Wang (1994) proposed $C$. sexmaculata mainly based on the presence of SC. However, we found this character to be quite common in populations of Henan, Gansu, Guangxi, Guizhou, and Sichuan (Figure 3d-h). Both cases (presence or absence of SC) can even appear in the same populations, e.g. Emeishan (Sichuan), Hechi (Guangxi) and Jinggangshan (Jiangxi) (Figure 3c, d). Another diagnostic character of $C$. sexmaculata is "the pale middle dorsal longitudinal stripe was continuous from S3 to S9" (Wang, 1994, p. 82). But the same character was also found in other populations, including Wenxian, Gansu. Furthermore, the first author XY has finally found the type specimens of $C$. sexmaculata from Shanghai Entomological Museum, Chinese Academy of Sciences (not the place Wang (1994) indicated). After checking the types, we confirmed that C. sexmaculata is a junior synonym of $C$. cyanomelas. 


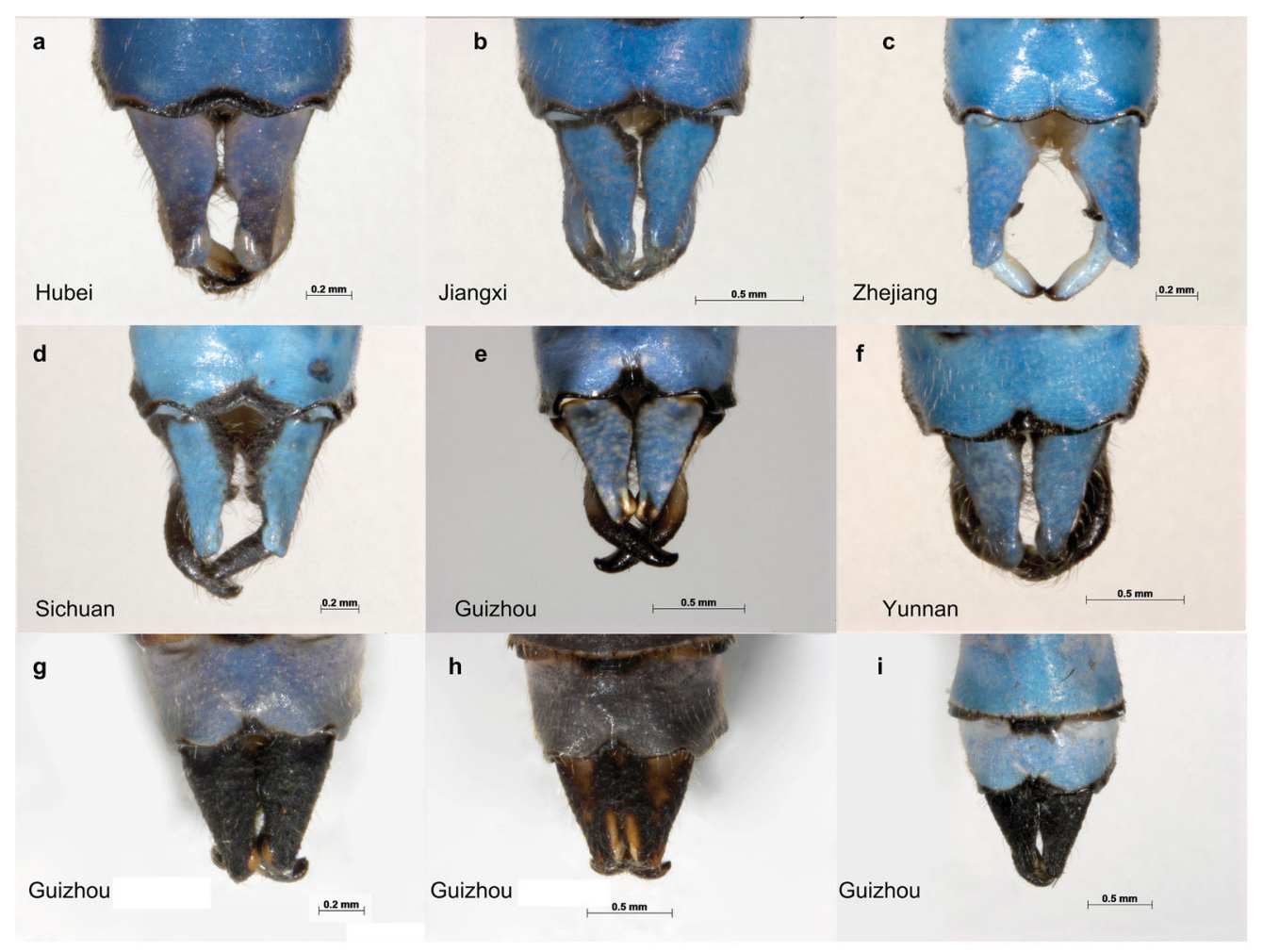

Figure 7. Dorsal view of caudal appendages of C. cyanomelas.

$\mathrm{Xu}$ (2006) proposed $C$. mingxiensis on the basis of a definite teneral male of Coeliccia species. According to his descriptions and figures, all characters of $C$. mingxiensis are identical to a teneral C. cyanomelas. Again, we have a large number of specimens covering the type locality of C. mingxiensis (Mingxi, located in Wuyi mountain, Fujian). After checking all these specimens (including some teneral ones) we believe that $C$. mingxiensis is just a junior synonym of C. cyanomelas. There is a long-standing confusion among Chinese workers about the teneral male color pattern of $C$. cyanomelas, which has even led to the irrelevant civil name "yellow stripe". Young males of $C$. cyanomelas always have an unusual but uniform yellow brown body color (Figure 3b, i), which becomes blue and decorated with a completely different pattern after maturing. Cuong, Thai, and Hong (2011) reported a new record of C. mingxiensis from Tam Dao National Park in north Vietnam. According to the photos of body and caudal appendages, the individuals they observed should all be teneral males of Coeliccia species (resemble cyanomelas), thus it is difficult to decide the exact species. If figures of genital ligula had been provided it would be helpful.

Zhang \& Huo (2011) published C. wilsoni on the basis of dry specimens collected in 2006, which had most probably had their blue body color decayed already (commonly in dry specimens of C. cyanomelas). According to wing vein and the 'fake' body color, Zhang thought this 'new species' is close to C. ryukyuensis, a completely different Japanese species. Generally, wing vein is not suitable for species level classification due to intraspecies variations caused by reasons such as fluctuating asymmetry (Hardersen, 2000). Zhang \& Huo (2011) emphasized that the shape of genital ligula of $C$. wilsoni was different from $C$. cyanomelas. This was because they had not compared enough specimens from other populations. We have found continuous variations in the shape of genital ligula of $C$. cyanomelas (Figure 8). In terms of the color pattern of 


\section{a}

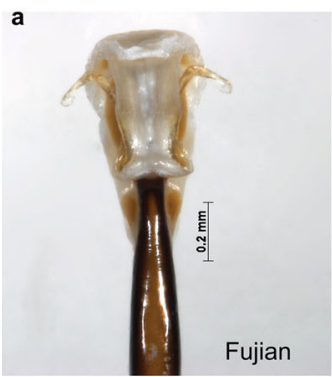

e

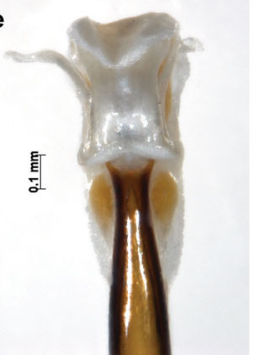

Sichuan b

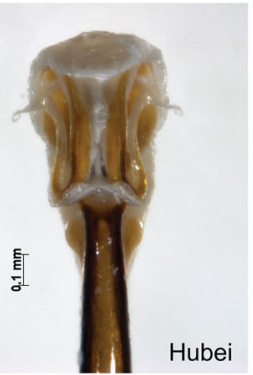

f

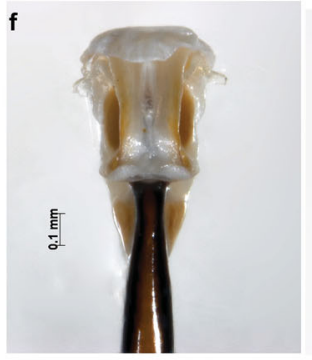

Zhejiang
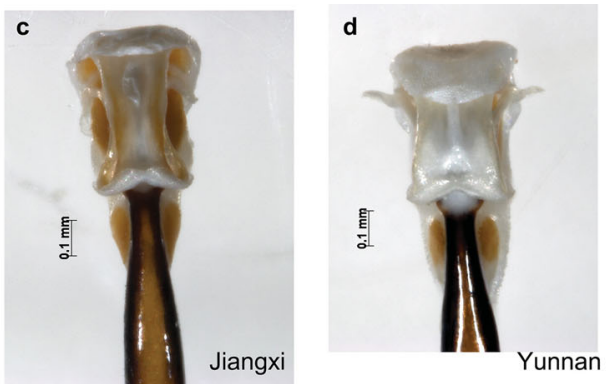

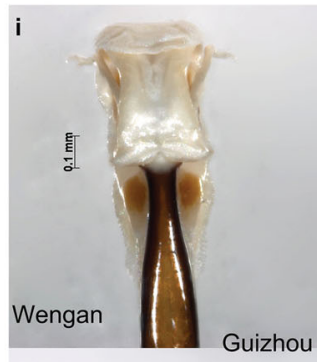

\section{j}
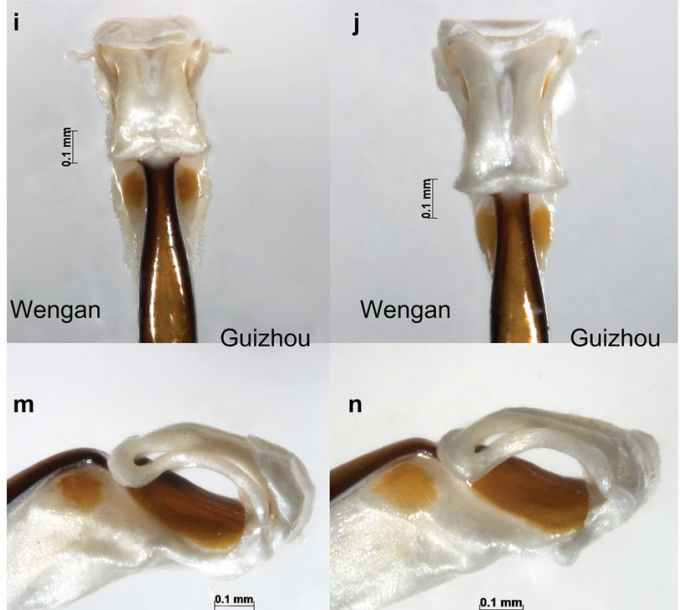

Wengan
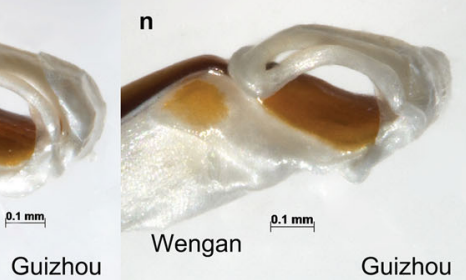

g

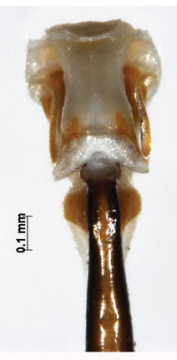

Hainan

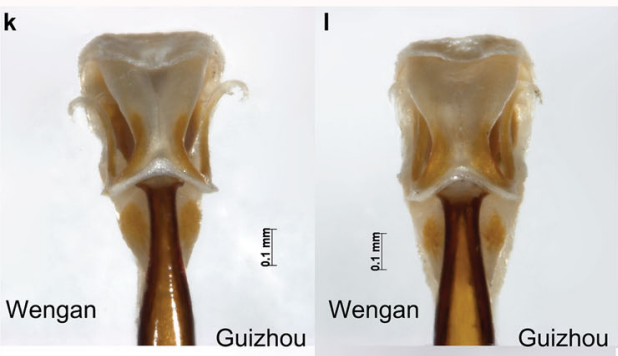

$\circ$

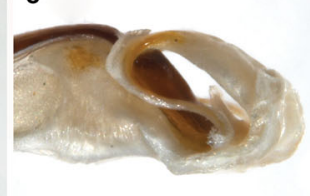

Wengan h

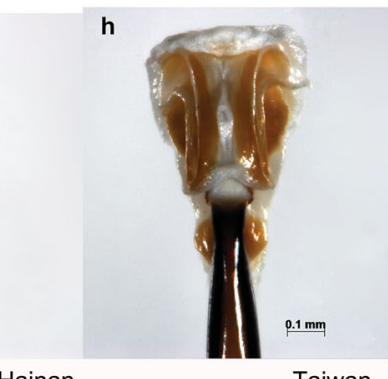

Taiwan

Figure 8. Genital ligulae of C. cyanomelas.

thorax and abdomen, as well as the shape of genital ligula, $C$. wilsoni should be the same species as $C$. cyanomelas. Furthermore, we have checked a series of specimens from the type locality (Nanzheng, Shaanxi) of $C$. wilsoni. Here we confirm that $C$. wilsoni should be a junior synonym of C. cyanomelas.

\section{New variety}

During fieldwork in Wengang, Libo, Guizhou we found an interesting little population of C. cyanomelas, of which the male color pattern is quite different from other populations 
(Figure $4 \mathrm{~g}-\mathrm{i}$ ). The pale color on compound eyes is green anteriorly and blue posteriorly in life (Figures 2, $4 \mathrm{~h}$ ) rather than wholly blue (Figures 3, 4a-f). Antehumeral stripes are small, similar size and shape to SS, pale blue with hint of green (Figure $4 \mathrm{~g}, \mathrm{~h}$ ); those are definitely larger than SS and wholly blue in other populations (Figures $3 \mathrm{c}-\mathrm{h}, 4 \mathrm{a}-\mathrm{f}$ ). Caudal appendages are wholly black (Figures $5 \mathrm{~g}, \mathrm{i}, 7 \mathrm{~g}$, i) rather than blue (Figures $5 \mathrm{a}-\mathrm{f}, 7 \mathrm{a}-\mathrm{f}$ ). Head of genital ligula is triangular (Figure $8 \mathrm{k}, 1$ ) but shield-like in the sympatric general population as well as most other populations (Figure 8i, j). However, just as we have discussed above, the shape of genital ligula has obvious variations among populations, therefore we will not emphasize this character here. The same also happens to the shape of caudal appendages. For example, we still cannot find any distinct gaps in the shape variations of the male cercus, although the detailed structure of it is complicated (Figure 6). The more individuals are studied the more difficult is in determining diagnostic differences.

The Wengang population was restricted to a very limited habitat, viz. at the foot of a huge precipice about $1 \mathrm{~km}$ long. Males occurred in moderate density, but very few females were observed. No individuals from the sympatric general population ( $c f$ Figure $3 \mathrm{f}$ ) were found within this range although they could be observed not far away. Preliminary molecular analysis (unpublished) showed that the Wengang population is not a distinct species. According to ICZN rules it cannot be a subspecies due to the sympatric distribution. We do not think it is a seasonal form either, since we are sure it will live simultaneously with the general population for quite a long time. Here we would like to treat this population as a Darwinian variety or incipient species which deserves further study.

\section{Acknowledgements}

We thank Dr. Weibing Zhu from Shanghai Entomological Museum, Chinese Academy of Sciences, for helping to check the type specimens. We are grateful to the reviewers and the editor for their valuable remarks and suggestions.

\section{Funding}

This project was supported by the grant of Ministry of Science and Technology of China (no. 2015FY210300), the National Natural Science Foundation of China (no. 31572299) and the Chongqing basic research and frontier exploration special project (no. cstc2018jcyjAX0415).

\section{References}

Asahina, S. (1956). Dragonflies of West Tien-Mu-Shan, central China. Entomologiske Meddelelser, 27, $204-228$.

Asahina, S. (1961). Contributions to the knowledge of the Odonata fauna of central China. Tombo, 4, 1-17.

Asahina, S. (1984). A list of the Odonata recorded from Thailand. Part VI. Platycnemididae-Genus Coeliccia. Tombo, 27(1-4), 1-20.

Cuong, D. O., Thai, N. Q., \& Hong, B. M. (2011, October). Dragonfly Coeliccia Mingxiensis Xu, 2006 (Odonata: Platyneminidea) from Tam Dao National Park with additional notes on its morphology. Proceedings of the Fourth National Science Congress on Ecology and Biological Resources, Hanoi, 68-69.

Fraser, F. C. (1933). The fauna of British India - Odonata. London: Taylor and Francis.

Hardersen, S. (2000). The role of behavioural ecology of damselflies in the use of fluctuating asymmetry as a bioindicator of water pollution. Ecological Entomology, 25, 45-53.

Needham, J. G. (1930). A manual of the dragonflies of China. Peiping: The Fan Memorial Institute of Biology.

Ris, F. (1912). Neue Libellen von Formosa, Südchina, Tonkin und den Philippinen. Supplementa entomologica, 1, 44-85.

Schorr, M., Lindeboom, M., \& Paulson, D. R. (2009). List of Odonata of the world (Part 1, Zygoptera and Anisozygoptera) [Online]. Retrieved from http://www.pugetsound.edu/academics/academic-resources/slater-museum/biodi versity-resources/dragonflies/world-odonata-list2

Steinhoff, P. O. M., \& Uhl, G. (2015). Taxonomy and nomenclature of some mainland SE-Asian Coeliccia species (Odonata, Platycnemididae) using micro-CT analysis. Zootaxa, 4059(2), 257-276. doi: 10.11646/zootaxa.4059.2.2

Sui, J. Z., \& Sun, H. G. (1986). Common species of dragonflies from China. Beijing: Agriculture Publishing House.

Wang, Z. G. (1994). A new species of Coeliccia (Odonata: Platycnemididae) from China. Entomotaxonomia, 16(2), $82-84$. 
Xu, Q. H. (2006). Coeliccia mingxiensis sp. nov. from Fujian, China (Odonata: Platycnemididae). International Journal of Odonatology, 9(2), 250-254.

Yu, X. (2008). A taxonomic study on the superfamilies Coenagrionoidea and Lestoidea (Odonata: Zygoptera) from China. Doctoral Dissertation, Nankai University, Tianjin, China.

Yu, X. (2010). Odonata Research [Online]. Retrieved from http://www.china-odonata.top

Yu, X., \& Bu, W. J. (2009). A revision of Mesopodagrion McLachlan, 1896 (Odonata: Zygoptera: Megapodagrionidae). Zootaxa, 2202, 59-68.

Zhang, H. J., \& Huo, K. K. (2011). A study of the genus Coeliccia Kirby, 1890 from Shaanxi (China), with the description of C. wilsoni Zhang \& Yang spec. nov. (Zygoptera: Platycnemididae). Odonatologica, 40(1), 51-56. 